\title{
Fixed Points of Fuzzy Soft Mappings
}

\author{
Mujahid Abbas ${ }^{1}$, Asma Khalid ${ }^{2}$ and Salvador Romaguera ${ }^{3, *}$ \\ ${ }^{1}$ Department of Mathematics and Applied Mathematics, University of Pretoria, Lynnwood road, Pretoria 0002, South Africa \\ 2 Department of Mathematics, Lahore University of Management Sciences, 54792 Lahore, Pakistan \\ ${ }^{3}$ Instituto Universitario de Matemática Pura y Aplicada, Universitat Politècnica de València, Camí de Vera s/n, 46022 Valencia, Spain
}

Received: 10 Aug. 2013, Revised: 7 Nov. 2013, Accepted: 8 Nov. 2013

Published online: 1 Sep. 2014

\begin{abstract}
In this paper, the concept of a fuzzy soft mapping on a fuzzy soft set is introduced and the study of fixed points of such mappings is initiated.
\end{abstract}

Keywords: Fuzzy soft set, fuzzy soft mapping, fixed point

\section{Introduction}

The concept of fuzzy soft set, introduced by Molodstov in [12], is a recent development to deal with uncertainties. The contribution made by probability theory, fuzzy set theory, vague sets, rough sets and interval mathematics to deal with uncertainty is of vital importance but the problem of inadequacy of parameters has been successfully resolved by Soft set theory. Maji et al. ([9], [10]) and Maji and Roy ([11]) elaborated on the theory of soft sets, fuzzy soft sets and intuitionistic fuzzy soft sets and highlighted some of their applications. Some basic operations of fuzzy soft union and intersection and other algebraic properties were studied by Ahmad and Kharal ([1]). Babitha and Sunil ([3]) and Sut ([16]) defined soft set relations and fuzzy soft relations and applied the theory to decision making problems. Biwas and Samanta ([6]) introduced relations on intuitionistic fuzzy soft sets.

The notion of soft topology on a soft set was introduced by Cagman et. al ([4]) and several properties of soft topological spaces have been discussed, among others, by Shabir and Naz ([15]), Hussain and Ahmad ([7]), and Chen ([5]). Fuzzy soft topological spaces were studied by Tridiv ([13]) and Mahanta ([8]).

Recently, Wardowski ([17]) introduced a notion of soft mapping and obtained its fixed point. Motivated by his work, we initiate the study of fixed point in fuzzy soft set theory. For this purpose we discuss some properties of a fuzzy soft element in Section 3 of this paper. In Section 4 we introduce fuzzy soft mappings with the help of cartesian product and relations on fuzzy soft sets.
Concepts of fuzzy soft elements and fuzzy soft mappings to study fixed point theorems in the framework of fuzzy soft topological spaces are introduced in Section 5. Section 6 concludes the paper and gives insight to some possible future work.

\section{Preliminaries}

Throughout this section, by $U, E$ and $P(U)$, we denote an initial universe, a set of parameters, and the collection of all subsets of $U$, respectively.

Definition 1.([18]) A fuzzy set $A$ in $U$ is characterized by a function with domain as $U$ and values in $[0,1]$. The collection of all fuzzy sets in $U$ is denoted by $I^{U}$.

Definition 2.([18]) An empty fuzzy set denoted by $\widetilde{0}$ is a function which maps each $x \in U$ to 0 . That is, $\widetilde{0}(x)=0$ for all $x \in U$. A universal fuzzy set denoted by $\widetilde{1}$ is a function which maps each $x \in U$ to 1 . That is, $\widetilde{1}(x)=1$ for all $x \in U$.

If $A, B \in I^{U}$ we write $A \preceq B$ whenever $A(x) \leq B(x)$ for each $x \in U$, and $A=B$ whenever $A \preceq B$ and $B \preceq A$ for all $x \in U$.

Definition 3. ([18]) Let $A$ and $B$ be two fuzzy sets. Then (a) their union $A \cup B$ is defined as $(A \cup B)(x)=\max \{A(x), B(x)\}$; (b) their intersection $A \cap B$ is defined as $(A \cap B)(x)=\min \{A(x), B(x)\}$, and $(c)$ difference of $B$ from $A$ is denoted by $A / B$ and is defined by $(A / B)(x)=A(x)-B(x)$ for all $x \in U$.

\footnotetext{
*Corresponding author e-mail: sromague@ mat.upv.es
} 
Note that an implicit assumption $B \preceq A$ has been imposed to make the operation $A / B$ well defined.

Definition 4. ([18]) The complement of a fuzzy set $A$ is denoted by $A^{c}$ and is defined by $A^{c}(x)=1-A(x)$.

Definition 5.([12]) If $F$ is a mapping on $E$ taking values in $P(U)$, then a pair $(F, E)_{s}$ is called a soft set over $(U, E)$.

Definition 6.([9]) Let $A$ be a subset of E. A pair $(F, A)$ is called a fuzzy soft set over $(U, E)$ if $F: A \rightarrow I^{U}$ is a mapping from A into $I^{U}$. The collection of all fuzzy soft sets over $(U, E)$ is denoted by $\mathscr{F}(U, E)$.

A fuzzy soft set $(F, A)$ over $(U, E)$ is said to be:

(a) null fuzzy soft set if for each $e \in A, F(e)$ is a null fuzzy set $\widetilde{0}$ over $U$. We denote it by $\widetilde{\Phi}$.

(b) absolute fuzzy soft set if for each $e \in A, F(e)$ is a fuzzy universal set $\widetilde{1}$ over $U$. We denote it by $\widetilde{E}$.

Definition 7.([9]) For two fuzzy soft sets $(F, A)$ and $(G, B)$ in $\mathscr{F}(U, E)$, we say that $(F, A) \widetilde{\subseteq}(G, B)$ if $A \subseteq B$ and $F(e) \preceq G(e)$ for each $e \in A$.

Definition 8.([9]) Two fuzzy soft sets $(F, A)$ and $(G, B)$ in $\mathscr{F}(U, E)$ are equal if $F \widetilde{\subseteq} G$ and $G \subseteq \widetilde{\simeq} F$.

Definition 9.([9]) The difference between two fuzzy soft sets $(F, E),(G, E)$ in $\mathscr{F}(U, E)$ is a fuzzy soft set $(\widetilde{F} / G, E)$ (say) defined by $(\widetilde{F / G})(e)=F(e) / G(e)$ for each $e \in E$.

Definition 10.([9]) The complement of a fuzzy soft set $(F, E)$ is a fuzzy soft set $\left(F^{\widetilde{c}}, E\right)$ defined by $F^{\widetilde{c}}(e)=\widetilde{1}$ / $F(e)$ for each $e \in E$.

$$
\text { Clearly } F^{\widetilde{c}}=\widetilde{E} / F, \widetilde{\Phi^{\widetilde{c}}}=\widetilde{E} \text {, and }\left((F)^{\widetilde{c}}\right)^{\widetilde{c}}=F \text {. }
$$

Definition 11.([1]) Let $(F, A)$ and $(G, B)$ be two fuzzy soft sets in $\mathscr{F}(U, E)$ with $A \cap B \neq \widetilde{\Phi}$, then (d) their intersection $(F \widetilde{\cap} G, C)$ is a fuzzy soft set, where $\mathrm{C}=A \cap B$ and, $(F \widetilde{\cap} G) e=F(e) \cap G(e)$ for each $e \in \mathrm{C}$, and $(e)$ their union $(F \widetilde{\cup} G, C)$ is a fuzzy soft set, where $\mathbf{C}=A \cup B$ and $(F \widetilde{\cup} G) e=F(e) \cup G(e)$ for each $e \in \mathbf{C}$.

Definition 12. ([14]) A fuzzy soft topology $\tau$ on $F \in \mathscr{F}(U, E)$ is a collection of fuzzy soft subsets of $F$ satisfying:

1. $\widetilde{\Phi}, F \in \tau$ ( this means that $\widetilde{E}$ is fuzzy soft subset of $F$, that is, $\widetilde{1}(e) \preceq F(e)$, that is $1 \leq F(e)(x)$

2.If $F_{1}, F_{2} \in \tau$ then $F_{1} \widetilde{\cap} F_{2} \in \tau$.

3.If $F_{\alpha} \in \tau$ for all $\alpha \in \Lambda$, with $\Lambda$ an index set, then $\widetilde{\cup}_{\alpha \in \Lambda} F_{\alpha} \in \tau$.

If $\tau$ is a fuzzy soft topology on $F$ then the pair $(F, \tau)$ is called a fuzzy soft topological space.

\section{Fuzzy soft elements}

Fuzzy soft element is defined as follows.

Definition 13. ([13], [8]) Let e be any element in a set $A \subseteq$ $E$. A fuzzy soft set $F$ over $A$ is called a fuzzy soft element if $F\left(e^{\prime}\right)$ is a null fuzzy set for each $e^{\prime} \in A-\{e\}$. We denote it by $\left(F^{e}, A\right)$ or simply by $F^{e}$

A fuzzy soft element $F^{e}$ is said to be in fuzzy soft set $(G, B)$ if $\left(F^{e}, A\right) \widetilde{\subseteq}(G, B)$. That is, $A \subseteq B$ and $F^{e}\left(e^{\prime}\right) \preceq G\left(e^{\prime}\right)$ for each $e^{\prime} \in A$, that is, $F^{e}(e) \preceq G\left(e^{\prime}\right)$ for each $e^{\prime} \in A$. We write it as $F^{e} \widetilde{\in} G$. It is straightforward to check that union of all fuzzy soft elements corresponding to each parameter $e \in A$ is equal to the approximate fuzzy soft set $F(e)$ and therefore the collection of all such unions, corresponding to each parameter, results in the original fuzzy soft set $(F, A)$.

Note that if $F$ is a fuzzy soft set in $\mathscr{F}(U, E)$ and $F^{e} \tilde{\in} F$ then $F=\left\{\widetilde{\cup}_{F e} \tilde{\in}_{F} F^{e}: e \in E\right\}$.

Example 1.Let $F$ be the fuzzy soft set in $\mathscr{F}(U, E)$ defined as

$$
F=\left\{\left(e_{1},\left\{\frac{u_{1}}{0.5}, \frac{u_{2}}{0.3}\right\}\right),\left(e_{2},\left\{\frac{u_{1}}{0.7}, \frac{u_{2}}{0.4}\right\}\right)\right\}
$$

Then some of the fuzzy soft elements of $F$ are

$F^{e_{1}}=\left\{\left(e_{1},\left\{\frac{u_{1}}{0.3}, \frac{u_{2}}{0.1}\right\}\right)\right\}, F^{\prime e_{1}}=\left\{\left(e_{1},\left\{\frac{u_{1}}{0.5}, \frac{u_{2}}{0.3}\right\}\right)\right\}$ and $F^{e_{2}}=\left\{\left(e_{2},\left\{\frac{u_{1}}{0.7}, \frac{u_{2}}{0.4}\right\}\right)\right\}$.

Note that $F^{e_{1}} \widetilde{\cup} F^{\prime e_{1}}=\left\{\left(e_{1},\left\{\frac{u_{1}}{0.5}, \frac{u_{2}}{0.3}\right\}\right)\right\}=F\left(e_{1}\right)$. Similarly,

$$
\widetilde{\cup} F^{e_{2}}=\left\{\left(e_{2},\left\{\frac{u_{1}}{0.7}, \frac{u_{2}}{0.4}\right\}\right)=F\left(e_{2}\right) .\right.
$$

Therefore, $\left\{\widetilde{\cup}_{F^{e_{1}} \in F} F^{e_{1}}, \widetilde{\cup}_{F^{e_{2}}} F^{e_{2}}\right\}=F$.

Basic properties with held by fuzzy soft elements are stated in the following proposition.

Proposition 1. Let $F_{1}, F_{2}$ be two fuzzy soft sets over $(U, E)$ and $e \in E$ The following holds.

1. $\widetilde{\Phi}$ is an empty fuzzy soft element of every fuzzy soft set.

2.If $F$ is a fuzzy soft set such that $F \neq \widetilde{\Phi}$, then $F$ contains at least one non empty fuzzy soft element.

3.If $F^{e} \widetilde{\in} F_{1} \widetilde{\cup} F_{2}$ then $F^{e}$ is a fuzzy soft element of $F_{1}$ or $F_{2}$.

4. $F^{e} \widetilde{\in} F_{1} \widetilde{\cap} F_{2}$ if and only if $F^{e}$ is a fuzzy soft element of $F_{1}$ and $F_{2}$.

5.If $F^{e} \widetilde{\in} F_{1} \backslash F_{2}$ then $F^{e}$ is a fuzzy soft element of $F_{1}$ but not necessarily a fuzzy soft element of $F_{2}$.

Proof. 1. Let $e$ be an element of $E$ and $F$ a fuzzy soft set over $E$. Obviously, $\widetilde{\Phi}(e) \preceq F(e)$ as $\widetilde{\Phi}(e)(x)=0$ for each $x \in U$. Therefore $\widetilde{\Phi}$ is an empty fuzzy soft element of every fuzzy soft set. 
2. If $F \neq \widetilde{\Phi}$, then there exists at least one $e^{*} \in E$ such that $F\left(e^{*}\right) \neq \widetilde{0}$, that is, there exists an $x \in U$ for which $F\left(e^{*}\right)(x) \neq 0$. Let $F\left(e^{*}\right)(x)=\varepsilon$ for some $\varepsilon \in(0,1]$. Then we define $F_{1}$ such that

$$
F_{1}\left(e^{*}\right)(x)=\frac{\varepsilon}{2} \text { and } F_{1}(e)(x)=0 \text { whenever } e \neq e^{*} .
$$

This implies that $F_{1}\left(e^{*}\right) \preceq F\left(e^{*}\right)$. If $e \neq e^{*}$, then $\widetilde{0}=F_{1}(e) \preceq F(e)$. Hence fuzzy soft set $F_{1}$ is a non empty fuzzy soft element of $F$.

3. Let $F^{e}$ be a fuzzy sof element of $F_{1} \widetilde{\cup} F_{2}$, that is, $F^{e} \widetilde{\in}\left(F_{1} \widetilde{\cup} F_{2}\right)$ which implies that $F^{e}(e) \preceq F_{1}\left(e^{\prime}\right) \cup F_{2}\left(e^{\prime}\right)$ for each $e^{\prime} \in E$. So, for each $x \in U$, $F^{e}(e)(x) \leq \max \left\{F_{1}\left(e^{\prime}\right)(x), F_{2}\left(e^{\prime}\right)(x)\right\}$. Now if $F_{1}\left(e^{\prime}\right)(x) \leq F_{2}\left(e^{\prime}\right)(x)$ then for each $e^{\prime} \in E$, $F^{e}(e) \preceq F_{2}\left(e^{\prime}\right)$. Hence $F^{e} \widetilde{\in} F_{2}$. If $F_{2}\left(e^{\prime}\right)(x) \leq F_{1}\left(e^{\prime}\right)(x)$ then $F^{e}(e) \preceq F_{1}\left(e^{\prime}\right)$ for each $e^{\prime} \in E$ which implies that $F^{e} \widetilde{\in} F_{1}$. So, $F^{e} \widetilde{\in} F_{1}$ or $F^{e} \widetilde{\in} F_{2}$. Conversely, suppose that $F^{e} \widetilde{\in} F_{1}$ or $F^{e} \widetilde{\in} F_{2}$. Then $F^{e}(e) \preceq F_{1}\left(e^{\prime}\right)$ or $F^{e}(e) \preceq F_{2}\left(e^{\prime}\right)$ for each $e^{\prime} \in E$, that is, for all $x \in U, F^{e}(e)(x) \leq F_{1}\left(e^{\prime}\right)(x)$ or $F^{e}(e)(x) \leq F_{2}\left(e^{\prime}\right)(x)$. Thus $F^{e}(e)(x)$ $\leq \max \left\{F_{1}\left(e^{\prime}\right)(x), F_{2}\left(e^{\prime}\right)(x)\right\}$. Therefore $F^{e} \widetilde{\in} F_{1} \widetilde{\cup} F_{2}$.

4. Let $F^{e} \widetilde{\in}\left(F_{1} \widetilde{\cap} F_{2}\right)$ which implies that $F^{e}(e) \preceq F_{1}\left(e^{\prime}\right) \cap$ $F_{2}\left(e^{\prime}\right)$ for each $e^{\prime} \in E$. So for each $x \in U$,

$$
F^{e}(e)(x) \leq \min \left\{F_{1}\left(e^{\prime}\right)(x), F_{2}\left(e^{\prime}\right)(x)\right\} .
$$

If $\quad F_{1}\left(e^{\prime}\right)(x) \leq F_{2}\left(e^{\prime}\right)(x) \quad$ then $F^{e}(e)(x) \leq F_{1}\left(e^{\prime}\right)(x) \leq F_{2}\left(e^{\prime}\right)(x)$ implies that $F^{e}$ is a fuzzy sof element of $F_{1}$ and $F_{2}$. Similarly if $F_{2}\left(e^{\prime}\right)(x) \leq F_{1}\left(e^{\prime}\right)(x) \quad$ then $F^{e}(e)(x) \leq F_{2}\left(e^{\prime}\right)(x) \leq F_{1}\left(e^{\prime}\right)(x)$ means that $F^{e}$ is a fuzzy sof element of $F_{2}$ and $F_{1}$. Conversely, suppose that $F^{e} \widetilde{\in} F_{1}$ and $F^{e} \widetilde{\in} F_{2}$. Then, for each $e^{\prime} \in E, F^{e}(e) \preceq F_{1}\left(e^{\prime}\right)$ and $F^{e}(e) \preceq F_{2}\left(e^{\prime}\right)$ which implies that

$$
F^{e}(e)(x) \leq \min \left\{F_{1}\left(e^{\prime}\right)(x), F_{2}\left(e^{\prime}\right)(x)\right\}
$$

for each $x$ in $U$. Therefore, $F^{e} \widetilde{\in} F_{1} \widetilde{\cap} F_{2}$.

5. Let $F^{e} \widetilde{\in} F_{1} \widetilde{\backslash} F_{2}$. Then, $F^{e}(e) \preceq F_{1}\left(e^{\prime}\right) \backslash F_{2}\left(e^{\prime}\right)$ for each $e^{\prime} \in E$, that is, $F^{e}(e)(x) \leq F_{1}\left(e^{\prime}\right)(x)-F_{2}\left(e^{\prime}\right)(x)$ for each $x \in U$. Then $F^{e}(e)(x) \leq F_{1}\left(e^{\prime}\right)(x)$ but the real number $F^{e}(e)(x)$ is not necessarily less than $F_{2}\left(e^{\prime}\right)(x)$ for each $x$. Therefore, $F^{e}$ is a fuzzy soft element of $F_{1}$ but $F^{e}$ is not necessarily a fuzzy soft element of $F_{2}$.

Example 2. Suppose that $U=\left\{u_{1}, u_{2}, u_{3}\right\}$ and $E=\left\{e_{1}, e_{2}\right\}$. Let $F$ and $G \in \mathscr{F}(U, E)$ be of the form $F=\left\{\left(e_{1},\left\{\frac{u_{1}}{0.6}, \frac{u_{2}}{0.8}, \frac{u_{3}}{0.3}\right\},\left(e_{2},\left\{\frac{u_{1}}{0.4}, \frac{u_{2}}{0.6}, \frac{u_{3}}{0.7}\right\}\right)\right\}\right.$ and $G=\left\{\left(e_{1},\left\{\frac{u_{1}}{0.5}, \frac{u_{2}}{0.8}, \frac{u_{3}}{0.3}\right\},\left(e_{2},\left\{\frac{u_{1}}{0.2}, \frac{u_{2}}{0.4}, \frac{u_{3}}{0.3}\right\}\right)\right\}\right.$.

Note that

$F \widetilde{\cup} G=\left\{\left(e_{1},\left\{\frac{u_{1}}{0.6}, \frac{u_{2}}{0.8}, \frac{u_{3}}{0.3}\right\},\left(e_{2},\left\{\frac{u_{1}}{0.4}, \frac{u_{2}}{0.6}, \frac{u_{3}}{0.7}\right\}\right)\right\}\right.$,

$F \widetilde{\cap} G=\left\{\left(e_{1},\left\{\frac{u_{1}}{0.5}, \frac{u_{2}}{0.8}, \frac{u_{3}}{0.3}\right\},\left(e_{2},\left\{\frac{u_{1}}{0.2}, \frac{u_{2}}{0.4}, \frac{u_{3}}{0.3}\right\}\right)\right\}\right.$, and $F \widetilde{\backslash} G=\left\{\left(e_{1},\left\{\frac{u_{1}}{0.1}\right\},\left(e_{2},\left\{\frac{u_{1}}{0.2}, \frac{u_{2}}{0.2}, \frac{u_{3}}{0.4}\right\}\right)\right\}\right.$.
F. $F^{e_{1}}=\left\{\left(e_{1},\left\{\frac{u_{1}}{0.4}, \frac{u_{2}}{0.1}, \frac{u_{3}}{0.3}\right\}\right)\right\}$ is a soft fuzzy element of

Note that $F^{e_{1}} \widetilde{\in} F \widetilde{\cup} G$. Similarly, $F^{e_{1}} \widetilde{\in} F \widetilde{\cap} G$. Also, $F^{e_{2}}=\left\{\left(e_{2},\left\{\frac{u_{1}}{0.1}, \frac{u_{2}}{0.1}, \frac{u_{3}}{0.4}\right\}\right)\right\}$ is a soft fuzzy point of $F \widetilde{\nearrow} G$ then $F^{e_{2}} \widetilde{\in} F$ but $F^{e_{2}}$ is not a fuzzy soft element of $G$.

Proposition 2. Let $F_{1}, F_{2}$ be two fuzzy soft sets over $E$. Then $F_{1} \widetilde{\subseteq} F_{2}$ if and only if $F^{e} \widetilde{\in} F_{1}$ implies that $F^{e} \widetilde{\in} F_{2}$.

Proof. Let $F_{1} \widetilde{\widetilde{\subseteq}} F_{2}$ then $F_{1}(e) \preceq F_{2}(e)$ for each $e \in E$, that is $F_{1}(e)(x) \leq F_{2}(e)(x)$ for each $x \in U$. Suppose that $F^{e} \widetilde{\in} F_{1}$. That is, for each $e^{\prime} \in E, F^{e}(e) \preceq F_{1}\left(e^{\prime}\right)$ and hence $F^{e}(e) \preceq F_{2}\left(e^{\prime}\right)$ for each $e^{\prime} \in E$. Therefore, $F^{e} \widetilde{\in} F_{2}$. Conversely, suppose that every fuzzy soft element $F^{e}$ in $F_{1}$ is also a fuzzy soft element of $F_{2}$. Let $\bar{F}_{1}^{e}$ to be the largest fuzzy soft element of $F_{1}$ for each $e \in E$ then $\bar{F}_{1}^{e} \widetilde{\in} F_{2}$. Let $\varepsilon \in(0,1]$ and $\bar{F}_{1}^{e}(e)(x)+\varepsilon$ be such that $\bar{F}_{1}^{e}(e)(x)+\varepsilon \leq F_{2}\left(e^{\prime}\right)(x)$ for each $x \in U$. That is, $\bar{F}_{1}^{e}(e)(x) \leq F_{2}\left(e^{\prime}\right)(x)$ for each $e^{\prime} \in E$. Therefore, $F_{1} \widetilde{\widetilde{C}} F_{2}$.

Definition 14.([8]) A fuzzy soft topological space $(F, \tau)$ is said to be a fuzzy soft Hausdorff space if for distinct fuzzy soft elements $F^{e}, F^{e^{\prime}}$ of $F$, there exists disjoint fuzzy soft open sets $\left(F_{1}, A\right)$ and $\left(F_{2}, A\right)$ such that $F^{e} \tilde{\in} F_{1}$ and $F^{e^{\prime}} \tilde{\in} F_{2}$.

Proposition 3.Let $(F, \tau)$ be a fuzzy soft topological space. A fuzzy soft set $V \widetilde{\subseteq} F$ is fuzzy soft open if and only if for each $F^{e} \widetilde{\in} V$ there exists a fuzzy soft set $W \widetilde{\in} \tau$ such that $F^{e} \widetilde{\in} W \widetilde{\subseteq} V$.

Proof. Let $V \in \tau$. Then clearly for each $F^{e} \tilde{\in} V$ we have $F^{e} \tilde{\in} V \widetilde{\subseteq} V$. Let $V \widetilde{\subseteq} F$ be such that for each $F^{e} \widetilde{\in} V$ there exists a fuzzy soft open set $W_{F^{e}}$ such that $F^{e} \widetilde{\in} W_{F} e \widetilde{\subseteq} V$ which means that $F^{e}(e) \preceq W_{F}\left(e^{\prime}\right) \preceq V\left(e^{\prime}\right)$ for each $e^{\prime} \in E$. Since for each $e \in E, V(e)=\widetilde{\cup}\left\{F^{e}: F^{e} \widetilde{\in} V\right\} \widetilde{\subseteq}$ $\widetilde{\cup} W_{F e}(e) \widetilde{\subseteq} V(e)$, we deduce that $V=\left\{\widetilde{\cup} W_{F e}: e \in E\right\} \in \tau$.

\section{Fuzzy soft mapping}

In this section, a concept of fuzzy soft mapping is introduced. Relevant definitions are formulated and some properties of fuzzy soft mappings are studied.

Definition 15.([2]) The cartesian product of two fuzzy soft sets $(F, A)$ and $(G, B)$ is defined as a fuzzy soft set $(H, C)=$ $(F, A) \hat{\times}(G, B)$, where $C=A \times B$ and $H: C \rightarrow \mathscr{F}(U, E)$ is defined by

$$
H\left(e, e^{\prime}\right)=F(e) \widetilde{\times} G\left(e^{\prime}\right)
$$

for all $\left(e, e^{\prime}\right) \in C$, where

$$
F(e) \widetilde{\times} G\left(e^{\prime}\right)=\left\{\frac{x}{\min \left\{F\left(e^{\prime}\right)(x), G\left(e^{\prime}\right)(x)\right.}: x \in U\right\} .
$$


Example 3. Let $U=\left\{u_{1}, u_{2}\right\}$ and $A=\left\{e_{1}, e_{2}, e_{3}\right\}$. Define fuzzy soft sets $F_{1}$ and $F_{2}$ as follows:

$$
\begin{aligned}
& \left(F_{1}, A\right)=\left\{\left(e_{1},\left\{\frac{u_{1}}{0.6}, \frac{u_{2}}{0.5}\right\}\right),\left(e_{2},\left\{\frac{u_{1}}{0.3}, \frac{u_{2}}{0.5}\right\}\right),\right. \\
& \left.\left(e_{3},\left\{\frac{u_{1}}{0.2}, \frac{u_{2}}{0.7}\right\}\right)\right\},
\end{aligned}
$$

and $\left.\left(e_{3},\left\{\frac{u_{1}}{0.5}, \frac{u_{2}}{0.4}\right\}\right)\right\}$.

$$
\left(F_{2}, A\right)=\left\{\left(e_{1},\left\{\frac{u_{1}}{0.3}, \frac{u_{2}}{0.4}\right\}\right),\left(e_{2},\left\{\frac{u_{1}}{0.6}, \frac{u_{2}}{0.7}\right\}\right),\right.
$$

Then $\left(F_{1}, A\right) \widehat{\times}\left(F_{2}, A\right)=(H, C)$ where $C=A \times A$ and $H$ is given by

$$
\begin{aligned}
& H\left(e_{1}, e_{1}\right)=F_{1}\left(e_{1}\right) \widetilde{\times} F_{2}\left(e_{1}\right)=\left\{\frac{u_{1}}{0.3}, \frac{u_{2}}{0.4}\right\}, \\
& H\left(e_{1}, e_{2}\right)=F_{1}\left(e_{1}\right) \widetilde{\times} F_{2}\left(e_{2}\right)=\left\{\frac{u_{1}}{0.6}, \frac{u_{2}}{0.5}\right\}, \\
& H\left(e_{1}, e_{3}\right)=F_{1}\left(e_{1}\right) \widetilde{\times} F_{2}\left(e_{3}\right)=\left\{\frac{u_{1}}{0.5}, \frac{u_{2}}{0.4}\right\}, \\
& H\left(e_{2}, e_{1}\right)=F_{1}\left(e_{2}\right) \widetilde{\times} F_{2}\left(e_{1}\right)=\left\{\frac{u_{1}}{0.3}, \frac{u_{2}}{0.4}\right\}, \\
& H\left(e_{2}, e_{2}\right)=F_{1}\left(e_{2}\right) \widetilde{\times} F_{2}\left(e_{2}\right)=\left\{\frac{u_{1}}{0.3}, \frac{u_{2}}{0.5}\right\}, \\
& H\left(e_{2}, e_{3}\right)=F_{1}\left(e_{2}\right) \widetilde{\times} F_{2}\left(e_{3}\right)=\left\{\frac{u_{1}}{0.3}, \frac{0.4}{u_{2}}\right\}, \\
& H\left(e_{3}, e_{1}\right)=F_{1}\left(e_{1}\right) \widetilde{\times} F_{2}\left(e_{1}\right)=\left\{\frac{u_{1}}{0.2}, \frac{u_{2}}{0.4}\right\}, \\
& H\left(e_{3}, e_{2}\right)=F_{1}\left(e_{1}\right) \widetilde{\times} F_{2}\left(e_{1}\right)=\left\{\frac{u_{1}}{0.2}, \frac{u_{2}}{0.7}\right\}, \\
& H\left(e_{3}, e_{3}\right)=F_{1}\left(e_{1}\right) \widetilde{\times} F_{2}\left(e_{1}\right)=\left\{\frac{u_{1}}{0.2}, \frac{u_{2}}{0.4}\right\} .
\end{aligned}
$$

Definition 16.Let $\left(F_{1}, A\right),\left(F_{2}, A\right)$ be fuzzy soft sets in $\mathscr{F}(U, E)$. A fuzzy soft set $R$ is called a fuzzy soft relation from $F_{1}$ to $F_{2}$ if $R=(G, D)$ where $D \subseteq C$ and $G=H$ on D.

Example 4.Let $F_{1}, F_{2}$ be as given in Example 3. Then

$$
R=\left\{F_{1}\left(e_{1}\right) \widetilde{\times} F_{2}\left(e_{2}\right), F_{1}\left(e_{2}\right) \widetilde{\times} F_{2}\left(e_{3}\right), F_{1}\left(e_{3}\right) \widetilde{\times} F_{2}\left(e_{3}\right)\right\}
$$

is a fuzzy soft relation from $F_{1}$ to $F_{2}$ which itself is a fuzzy soft set with $\left\{\left(e_{1}, e_{1}\right),\left(e_{2}, e_{3}\right),\left(e_{3}, e_{3}\right)\right\}$ as a set of parameters. By $F_{1} R F_{2}$, we mean that $F_{1}\left(e_{1}\right) \widetilde{\times} F_{2}\left(e_{2}\right) \in R$.

We now introduce a fuzzy soft mapping.

Definition 17. Let $F, G$ be fuzzy soft sets in $\mathscr{F}(U, E)$. A fuzzy soft relation $T$ from $F$ to $G$ is called a fuzzy soft mapping from $F$ to $G$ denoted by $T: F \rightarrow G$ if the following conditions are satisfied.

$\mathrm{C} 1$ for each fuzzy soft element $F^{e} \tilde{\in} F$, there exists only one fuzzy soft element $G^{e} \tilde{\in} G$ such that $F^{e} T G^{e}$ which will be denoted as $T\left(F^{e}\right)=G^{e}$.

C2for each fuzzy soft empty element $F^{e} \widetilde{\in} F, T\left(F^{e}\right)$ is a empty fuzzy soft element of $G$.
Definition 18.Let $F, G$ be fuzzy soft sets in $\mathscr{F}(U, E)$ and $T: F \rightarrow G$ a fuzzy soft mapping. The image of $X \subseteq F$ under fuzzy soft mapping $T$ is the fuzzy soft set $T(X)$ defined by

$$
T(X)=\left\{\widetilde{\cup}_{F^{e} \tilde{\in} X} T\left(F^{e}\right): e \in E\right\} .
$$

It is clear that $T(\widetilde{\Phi})=\widetilde{\Phi}$ for each fuzzy soft mapping $T$.

Definition 19. Let $F, G \in \mathscr{F}(U, E)$ and $T: F \rightarrow G$ a fuzzy soft mapping. The inverse image of $Y \widetilde{\widetilde{G}}$ under fuzzy soft mapping $T$ is the fuzzy soft set denoted by $T^{-1}(Y)$ and defined as:

$T^{-1}(Y)=\left\{\left\{\widetilde{\cup}_{F^{e} \tilde{\in} F} F^{e}: e \in E\right\}: T\left(F^{e}\right) \widetilde{\in} Y\right.$ for each $\left.e \in E\right\}$.

Example 5.Let $F$ and $G$ be defined as:

$F=\left\{\left(e_{1},\left\{\frac{u_{1}}{0.6}, \frac{u_{2}}{0.4}\right\}\right),\left(e_{2},\left\{\frac{u_{1}}{0.3}, \frac{u_{2}}{0.7}\right\}\right)\right\}$ and

$G=\left\{\left(e_{1},\left\{\frac{u_{1}}{0.2}, \frac{u_{2}}{0.6}\right\}\right),\left(e_{2},\left\{\frac{u_{1}}{0.7}, \frac{u_{2}}{0.8}\right\}\right)\right\}$.

Define $T$ as $T\left(F^{e}\right)=\widehat{G^{e}}$ for each $e \in E$, where $\widehat{G^{e}}$ is the largest fuzzy soft element corresponding to each parameter $e \in E$, that is, if $G^{e}$ is any fuzzy soft element in $G$ then $G^{e} \widetilde{\subseteq} \widehat{G}^{e}$. So, $T\left(F^{e_{1}}\right)=\widehat{G^{e_{1}}}=\left\{\frac{u_{1}}{0.2}, \frac{u_{2}}{0.6}\right\}$ for all $F^{e_{1}} \widetilde{\in} F$ and $T\left(F^{e_{2}}\right)=\widehat{G^{e_{2}}}=\left\{\frac{u_{1}}{0.7}, \frac{u_{2}}{0.8}\right\}$ for all $F^{e_{2}} \tilde{\in} F$. Moreover,

$$
\begin{aligned}
T(F) & =\left\{\left\{\cup_{F^{e_{1}} \tilde{\in X}} T\left(F^{e_{1}}\right)\right\},\left\{\cup_{F^{e_{2}} \tilde{\in} X} T\left(F^{e_{2}}\right)\right\}\right\} \\
& =\left\{\widehat{G^{e_{1}}}, \widehat{G^{e_{2}}}\right\}=G .
\end{aligned}
$$

Proposition 4. Let $F, \quad G \in \mathscr{F}(U, E)$, $(X, E),\left(X_{1}, E\right),\left(X_{2}, E\right) \quad \widetilde{\subseteq}(F, E), \quad$ and $(Y, E),\left(Y_{1}, E\right),\left(Y_{2}, E\right) \widetilde{\subseteq}(G, E)$. Let $T: F \rightarrow G$ be a fuzzy soft mapping. Then following hold.

i. $X_{1} \widetilde{\widetilde{\subseteq}} X_{2} \Rightarrow T\left(X_{1}\right) \widetilde{\simeq} T\left(X_{2}\right)$,

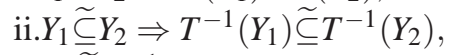

iii. $X \widetilde{\simeq} T^{-1}(T(X))$,

iv. $T\left(T^{-1}(Y)\right) \widetilde{\subseteq} Y$,

v. $T\left(X_{1} \widetilde{\cup} X_{2}\right)=T\left(X_{1}\right) \widetilde{\cup} T\left(X_{2}\right)$,

$\operatorname{vi.} T\left(X_{1} \widetilde{\cap} X_{2}\right)=T\left(X_{1}\right) \widetilde{\cap} T\left(X_{2}\right)$,

vii. $T^{-1}\left(Y_{1} \widetilde{\cup} Y_{2}\right)=T^{-1}\left(Y_{1}\right) \widetilde{\cup} T^{-1}\left(Y_{2}\right)$, and viii. $T^{-1}\left(Y_{1} \widetilde{\cap} Y_{2}\right)=T^{-1}\left(Y_{1}\right) \widetilde{\cap} T^{-1}\left(Y_{2}\right)$.

Proof. i. Let $F^{e}$ be an arbitrary fuzzy soft element in $T\left(X_{1}\right)$ then there exists a fuzzy soft element $\digamma^{e}$ in $X_{1}$ such that $T\left(\digamma^{e}\right)=F^{e}$. As $X_{1} \widetilde{\subseteq} X_{2}$ so $\digamma^{e}$ is a fuzzy soft element of $X_{2}$. So for every fuzzy soft element $F^{e}$ in $T\left(X_{1}\right), F^{e}$ is a fuzzy soft element in $T\left(X_{2}\right)$. Hence the result.

v. Let $\digamma^{e} \tilde{\in} T\left(X_{1} \widetilde{\cup} X_{2}\right)$. Then $\digamma^{e}=T\left(F^{e}\right)$ for some $F^{e} \tilde{\in} X_{1} \widetilde{\cup} X_{2}$. If $F^{e} \widetilde{\in} X_{1}$ then $\digamma^{e} \widetilde{\in} T\left(X_{1}\right) \widetilde{\simeq} T\left(X_{1}\right) \widetilde{\cup} T\left(X_{2}\right)$ and if $F^{e} \widetilde{\in} X_{2}$ then $\digamma^{e} \widetilde{\in} T\left(X_{2}\right) \widetilde{\subseteq} T\left(X_{1}\right) \widetilde{\cup} T\left(X_{2}\right)$. Therefore, $T\left(X_{1} \widetilde{\cup} X_{2}\right) \widetilde{\subseteq} T\left(X_{1}\right) \widetilde{\cup} T\left(X_{2}\right)$. Now let $\digamma^{e} \widetilde{\in} T\left(X_{1}\right) \widetilde{\cup} T\left(X_{2}\right)$, that is, $\digamma^{e}$ is fuzzy soft element of $T\left(X_{1}\right)$ or $T\left(X_{2}\right)$. If $F^{e} \widetilde{\in} T\left(X_{1}\right)$, then $T\left(X_{1}\right) \widetilde{\simeq} T\left(X_{1} \widetilde{\cup} X_{2}\right)$ gives $F^{e} \widetilde{\in} T\left(X_{1} \widetilde{\cup} X_{2}\right)$. 
Similarly, If $F^{e} \widetilde{\in} T\left(X_{2}\right)$, then $T\left(X_{2}\right) \widetilde{\subseteq} T\left(X_{1} \widetilde{\cup} X_{2}\right)$ gives $F^{e} \widetilde{\in} T\left(X_{1} \widetilde{\cup} X_{2}\right)$. Therefore $T\left(X_{1}\right) \widetilde{\cup} T\left(X_{2}\right) \widetilde{\subseteq} T\left(X_{1} \widetilde{\cup} X_{2}\right)$. So we conclude that

$$
T\left(X_{1} \widetilde{\cup} X_{2}\right)=T\left(X_{1}\right) \widetilde{\cup} T\left(X_{2}\right) .
$$

viii. If $F^{e} \widetilde{\in} T^{-1}\left(Y_{1} \widetilde{\cap} Y_{2}\right)$ then $T\left(F^{e}\right) \widetilde{\in} Y_{1} \widetilde{\cap} Y_{2}$. Since for each $e \in E, T\left(F^{e}\right) \widetilde{\subseteq} Y_{1}(e) \cap Y_{2}(e)$,then, for all $x, T\left(F^{e}\right)(x)$ is less than the minimum of $Y_{1}(e)(x)$ and $Y_{2}(e)(x)$. Hence, $F^{e} \widetilde{\in} T^{-1}\left(Y_{1}\right) \widetilde{\cap} T^{-1}\left(Y_{2}\right)$ and therefore,

$$
T^{-1}\left(Y_{1} \widetilde{\cap} Y_{2}\right) \widetilde{\subseteq} T^{-1}\left(Y_{1}\right) \widetilde{\cap} T^{-1}\left(Y_{2}\right) .
$$

Now, let $F^{e} \widetilde{\in} T^{-1}\left(Y_{1}\right) \widetilde{\cap} T^{-1}\left(Y_{2}\right)$. Then following similar arguments to those given above it follows that $T\left(F^{e}\right) \tilde{\in} Y_{1}$ and $T\left(F^{e}\right) \tilde{\in} Y_{2}$. It follows from here that $F^{e} \widetilde{\in} T^{-1}\left(Y_{1} \widetilde{\cap} Y_{2}\right)$. So, $T^{-1}\left(Y_{1}\right) \widetilde{\cap} T^{-1}\left(Y_{2}\right) \widetilde{\subseteq} T^{-1}\left(Y_{1} \widetilde{\cap} Y_{2}\right)$. lines.

Proofs of the rest of the properties follow on similar

Definition 20.Let $(F, \tau)$ be a fuzzy soft topological space and $K \widetilde{\subseteq} F$. A fuzzy soft open cover for $K$ is a collection of fuzzy soft open sets $\left\{V_{i}\right\}_{i \in I} \subseteq \tau$ whose union contains $K$.

Definition 21.A fuzzy soft topological space $(F, \tau)$ is compact if for each fuzzy soft open cover $\left\{V_{i}\right\}_{i \in I}$ of $K$ there exists $i_{1}, i_{2}, . ., i_{k} \in I, k \in \mathbb{N}$ such that $K \widetilde{\subseteq} \cup_{n=1}^{k} V_{i_{n}}$.

Definition 22.Let $(F, \tau),(G, v)$ be fuzzy soft topological spaces and $T: F \rightarrow G$ a fuzzy soft mapping. Then $T$ is a fuzzy soft continuous mapping (with respect to the fuzzy soft topologies $\tau$ and $v$ ) if for each $V \in v, T^{-1}(V) \in \tau$, that is, the inverse image of a fuzzy soft open set is a fuzzy soft open set.

We say that the fuzzy soft set $K \widetilde{\subseteq} F$ is fuzzy soft compact in $(F, \tau)$ if the fuzzy soft topological space $\left(K, \tau_{\mid K}\right)$ is fuzzy soft compact.

Example 6.Let $U=\left\{u_{1}, u_{2}, u_{3}\right\}, E=\left\{e_{1}, e_{2}, e_{3}\right\}$. Suppose $F \in \mathscr{F}(U, E)$ is of the form

$$
F=\left\{\left(e_{1},\left\{\frac{u_{1}}{1}, \frac{u_{2}}{1}, \frac{u_{3}}{0.7}\right\}\right),\left(e_{2},\left\{\frac{u_{1}}{0.6}, \frac{u_{2}}{0.9}, \frac{u_{3}}{0.7}\right\}\right)\right\} .
$$

Consider the family $\tau$ of all fuzzy soft subsets of $F$ and let $V=\widehat{F^{e_{1}}} \in \tau$ where $\widehat{F^{e_{1}}}$ is the largest fuzzy soft element of $F$. Define $T: F \rightarrow F$ as $T\left(F^{e}\right)=F^{e}$ for each $e \in E$. Then, $T^{-1}\left(\widehat{F^{e_{1}}}\right)=\widehat{F^{e_{1}}} \in \tau$.

Proposition 5.Let $(K, \tau)$ be a fuzzy soft compact topological space and $T: K \rightarrow K$ a fuzzy soft continuous mapping. Then $T(K)$ is a fuzzy soft compact set in $(K, \tau)$.

Proof. Suppose that $T(K) \widetilde{\widetilde{\cup}} \widetilde{\cup}_{\ell} G_{\ell}$, where $\left\{G_{\ell}\right\}$ is a family of fuzzy soft open sets in $K$. Then taking the preimage, we have, $K \widetilde{\subseteq} T^{-1}\left(\widetilde{\cup}_{\ell} G_{\ell}\right)$. As $T^{-1}\left(G_{\ell}\right)$ is open in $K$ so there must exist soft fuzzy open $V_{\ell} \widetilde{\subseteq} T(K)$ such that $T^{-1}\left(G_{\ell}\right)=V_{\ell} \widetilde{\cap} K$. So $K \widetilde{\subseteq} \widetilde{\cup}_{\ell}\left(V_{\ell} \widetilde{\cap} K\right)$ implies that $K \widetilde{\subseteq} \widetilde{\cup}_{\ell} V_{\ell}$. Since $K$ is compact fuzzy soft set, therefore there exist $\ell_{1}, \ell_{2}, \ldots, \ell_{N}$ such that $K \widetilde{\subseteq} \widetilde{\cup}_{i=1}^{N} V_{\ell_{i}}$. Hence $K=\widetilde{\cup}_{\ell}\left(V_{\ell} \widetilde{\cap} K\right)$ $=\widetilde{\cup}_{i=1}^{N} T^{-1}\left(G_{\ell_{i}}\right)$ which implies that $T(K) \widetilde{\widetilde{\subseteq}} \widetilde{\cup}_{i=1}^{N} G_{\ell_{i}}$. Hence $T(K)$ is compact.

\section{Fixed points of soft fuzzy mappings}

We start this section with the definition of a fixed point of a fuzzy soft mapping.

Definition 23.Let $F \in \mathscr{F}(U, E)$ be a fuzzy soft set and $T$ : $F \rightarrow F$ a fuzzy soft mapping. A fuzzy soft element $F^{e} \widetilde{\in} F$ is called a fixed point of $T$ if $T\left(F^{e}\right)=F^{e}$.

Example 7.If $T: F \rightarrow F$ is defined as an identity map, then each fuzzy soft element of $F$ is a fixed point.

Proposition 6.Let $(F, \tau)$ be a fuzzy soft compact topological space and $\left\{F_{n}: n \in \mathbb{N}\right\}$ a family of fuzzy soft subsets of $F$ satisfying:

A $1 . F_{n} \neq \widetilde{\Phi}$ for each $n \in \mathbb{N}$,

A2. $F_{n}$ is fuzzy soft closed for each $n \in \mathbb{N}$,

A3. $F_{n+1} \widetilde{\widetilde{\subseteq}} F_{n}$ for each $n \in \mathbb{N}$.

$$
\text { Then } \widetilde{\cap}_{n \in \mathbb{N}} F_{n} \neq \widetilde{\Phi} \text {. }
$$

Proof. Suppose on the contrary, that $\widetilde{\cap}_{n \in \mathbb{N}} F_{n}=\widetilde{\Phi}$. We know that $\left(\widetilde{\cap}_{n \in \mathbb{N}} F_{n}\right)^{\widetilde{c}}=\widetilde{\cup}_{n \in \mathbb{N}}\left(F_{n}\right)^{\widetilde{c}}($ see [1]). From $(A 2)$, $\left(F_{n}\right)^{\widetilde{c}}$ is a fuzzy soft open set for each $n \in \mathbb{N}$. Hence

$$
F \widetilde{\subseteq} \widetilde{E}=(\widetilde{\Phi})^{\widetilde{c}}=\left(\widetilde{\cap}_{n \in \mathbb{N}} F_{n}\right)^{\widetilde{c}}=\widetilde{\cup}_{n \in \mathbb{N}}\left(F_{n}\right)^{\widetilde{c}} .
$$

As $F$ is fuzzy soft compact, there exists $i_{1}, i_{2}, \ldots, i_{k} \in \mathbb{N}$, $i_{1}<i_{2}<\ldots<i_{k}, k \in \mathbb{N}$ such that

$$
F \widetilde{\subseteq} F_{i_{1}}^{\widetilde{c}} \widetilde{\cup} F_{i_{2}}^{\widetilde{c}}, \ldots \widetilde{\cup} F_{i_{k}}^{\widetilde{c}} .
$$

Hence from (A3), we have, $F_{i_{k}} \widetilde{\widetilde{C}} F \widetilde{\subseteq}\left(F_{i_{1}} \widetilde{\cap} F_{i_{2}} \widetilde{\cap} \text {... } \widetilde{\cap} F_{i_{k}}\right)^{\widetilde{c}}=$ $F_{i_{k}}^{\widetilde{c}}=\widetilde{E} / F_{i_{k}}$, which is impossible in the light of $(A 1)$.

Example 8.Let $(F, \tau)$ be a fuzzy soft topological space where $\tau$ contains all possible subsets of

$$
F=\left\{\left(e_{1},\left\{\frac{u_{1}}{1}, \frac{u_{2}}{0.7}\right\}\right),\left(e_{2},\left\{\frac{u_{1}}{0.9}, \frac{u_{2}}{1}\right\}\right)\right\} .
$$

Let two fuzzy soft subsets of $F$ be defined as

$$
F_{1}=\left\{\left(e_{1},\left\{\frac{u_{1}}{0.4}, \frac{u_{2}}{0.5}\right\}\right),\left(e_{2},\left\{\frac{u_{1}}{0.8}, \frac{u_{2}}{0.4}\right\}\right)\right\}
$$

and

$$
F_{2}=\left\{\left(e_{1},\left\{\frac{u_{1}}{0.6}, \frac{u_{2}}{0.3}\right\}\right),\left(e_{2},\left\{\frac{u_{1}}{0.8}, \frac{u_{2}}{0.5}\right\}\right)\right\} .
$$

Note that they satisfy the conditions of Proposition 6. Moreover $F_{1} \widetilde{\subseteq} F_{2}$ and $\widetilde{\cap}_{j=1}^{2} F_{j}=F_{1} \neq \widetilde{\Phi}$.

Proposition 7.Let $(F, \tau)$ be a fuzzy soft topological space and $T: F \rightarrow F$ a fuzzy soft mapping such that for each nonempty fuzzy soft element $F^{e} \widetilde{\in} F, T\left(F^{e}\right)$ is a nonempty fuzzy soft element of $F$. If $\widetilde{\cap}_{n \in \mathbb{N}} T^{n}(F)$ contains only one nonempty fuzzy soft element $F^{e} \widetilde{\in} F$, then $F^{e}$ is a unique fixed point of $T$. 
Proof. Observe that $T^{n}(F) \widetilde{\widetilde{\subseteq}} T^{n-1}(F)$ for each $n \in \mathbb{N}$. Let $F^{e}$ be a fuzzy soft element of $F$ such that $F^{e} \widetilde{\in} \widetilde{\cap}_{n \in \mathbb{N}} T^{n}(F)$. That is, $F^{e} \widetilde{\widetilde{\subseteq}} \widetilde{\cap}_{n \in \mathbb{N}} T^{n}(F)$. Consequently

$T\left(F^{e}\right) \widetilde{\subseteq} T\left(\widetilde{\cap}_{n \in \mathbb{N}} T^{n}(F)\right) \widetilde{\widetilde{\subseteq}} \widetilde{\cap}_{n \in \mathbb{N}} T^{n+1}(F) \widetilde{\widetilde{\cap}} \widetilde{\cap}_{n \in \mathbb{N}} T^{n}(F)=F^{e}$.

Since $T\left(F^{e}\right)$ is a non empty fuzzy soft element of $F$, therefore we obtain that $T\left(F^{e}\right)=F^{e}$.

Example 9.Let $(F, \tau)$ be a fuzzy soft topological space and define $T: F \rightarrow F$ as $T\left(F^{e}\right)=\widehat{F^{e}}$ for all $F^{e} \widetilde{\in} F$, where $F \neq$ $\widetilde{\Phi}$ and $\widehat{F^{e}}$ represents the largest fuzzy soft element of $F$ or equivalently $F^{e} \widetilde{\widetilde{C} F^{e}}$ for each fuzzy soft element $F^{e} \widetilde{\in} F$. Then $\widetilde{\cap}_{n \in \mathbb{N}} T^{n}(F)$ contains only one non empty fuzzy soft element which is $\widehat{F^{e}}$. Note that $\widehat{F^{e}}$ is a unique fixed point of $T$.

Proposition 8.Let $(F, \tau)$ be a fuzzy soft Hausdorff topological space. Then every fuzzy soft compact set in $F$ is fuzzy soft closed in $F$.

Proof. Let $K$ be a fuzzy soft compact set in $(F, \tau)$. We need to show that $K$ is fuzzy soft closed, that is, $K^{\widetilde{c}}$ is fuzzy soft open. Let $F^{e} \widetilde{\in} K^{\widetilde{c}}$. For every $F^{e^{\prime}} \widetilde{\in} K$, let $U_{i}, V_{i} \in \tau$ be such that $U_{i} \widetilde{\cap} V_{i}=\widetilde{\Phi}$ and $F^{e} \widetilde{\in} U_{i}, F^{e^{\prime}} \widetilde{\in} V_{i}$ where $i \in I$. Since $K$ is fuzzy soft compact so there exists $F^{e^{\prime}}, F^{e^{\prime}}, \ldots, F^{e^{\prime}} \widetilde{\in} K$ such that $K \widetilde{\subseteq} V_{i_{1}} \widetilde{\cup} V_{i_{2}} \widetilde{\cup} \ldots \widetilde{\cup} V_{i_{k}}$. Denote $U=U_{i_{1}} \widetilde{\cup} U_{i_{2}} \widetilde{\cup} \ldots \widetilde{\cup} U_{i_{k}}$ and $V=V_{i_{1}} \widetilde{\cup} V_{i_{2}} \widetilde{\cup} \ldots \widetilde{\cup} V_{i_{k}}$. Then $F^{e} \widetilde{\in} U \in \tau, U \widetilde{\cap} V=\widetilde{\Phi}$, which gives that $F^{e} \widetilde{\in} U \widetilde{\subseteq} K^{\widetilde{c}}$. Therefore $K$ is fuzzy soft closed.

Theorem 1.Let $(K, \tau)$ be a fuzzy soft compact Hausdorff topological space and $T: K \rightarrow K$ a fuzzy soft continuous mapping such that

a.for each non empty fuzzy soft element $F^{e} \widetilde{\in} K, T\left(F^{e}\right)$ is a non empty fuzzy soft element of $K$,

b.for each fuzzy soft closed set $X \widetilde{\widetilde{\subseteq}} K$ if $T(X)=X$ then $X$ contains only one nonempty fuzzy soft element of $K$.

Then there exists a unique nonempty fuzzy soft element $F^{e} \widetilde{\in} K$ such that $T\left(F^{e}\right)=F^{e}$.

Proof. Consider a family of fuzzy soft subsets of $K$ of the form

$C_{1}=T(K), C_{2}=T\left(C_{1}\right)=T^{2}(K), \ldots, C_{n}=T\left(C_{n-1}\right)=T^{n}(K)$

for $n \in \mathbb{N}$. It is clear that $C_{n} \widetilde{\widetilde{\subseteq}} C_{n-1}$ for each $n \in \mathbb{N}$. By Proposition 8, for each $n \in \mathbb{N}, C_{n}$ is fuzzy soft closed. Using Proposition 6, we conclude that a fuzzy soft set $D$ of the form $D=\cap_{n \in N} C_{n}$ is nonempty. Observe that

$T(D)=T\left(\cap_{n \in N} T^{n}(K)\right) \widetilde{\widetilde{C}} \cap_{n \in N} T^{n+1}(K) \widetilde{\widetilde{\subseteq}} \cap_{n \in N} T^{n}(K)=D$.

Now we show that $D \subseteq \widetilde{\simeq} T(D)$. For this, suppose that there exists $F^{e} \widetilde{\in} D$ such that $F^{e}$ is not a fuzzy soft element of $T(D)$. Put $E_{n}=T^{-1}\left(F^{e}\right) \widetilde{\cap} C_{n}$. Let us observe that $E_{n} \neq \widetilde{\Phi}$ and $E_{n} \widetilde{\widetilde{C}} E_{n-1}$ for each $n \in \mathbb{N}$. By Proposition 6 , there exists nonempty fuzzy soft element $\digamma^{e^{\prime}} \tilde{\in} T^{-1}\left(F^{e}\right) \widetilde{\cap} D$ and thus $F^{e}=T\left(\digamma^{e^{\prime}}\right) \tilde{\in} T(D)$, a contradiction. Therefore, $T(D)=D$. Hence the result follows using Proposition 6 .

\section{Conclusion}

In this paper we put forward the notion of fuzzy soft mappings based on the theory of fuzzy soft element of fuzzy soft set and fuzzy soft topological space. We study fixed points of fuzzy soft mappings. Employing these results, we can further study fixed point theory in the framework of fuzzy soft set theory.

Acknowledgement. The third author thanks the support of the Ministry of Economy and Competitiveness of Spain, under grant MTM2012-37894-C02-01.

\section{References}

[1] B. Ahmad and A. Kharal, On fuzzy soft sets, Adv. Fuzzy Syst., 2, 1-6 (2009).

[2] M. I. Ali and M. Shabir, Comments on De Morgan's law in fuzzy soft sets, Int. J. Fuzzy Math., 18, 679-686 (2010).

[3] K.V. Babitha, J.J. Sunil, Soft set relations and functions, Comput. Math. Appl., 60, 1840-1849 (2010).

[4] N. Cagman, S. Karatas, S. Enginoglu, Soft topology, Comput. Math. Appl., 62, 351-358 (2011).

[5] B. Chen, Soft semi-open sets and related properties in soft topological spaces, Appl. Math. Inf. Sci., 7, 287-294 (2013).

[6] B. Dinda and T.K. Samanta, Relations on intuitionistic fuzzy soft sets, Gen. Math. Notes, 1, 74-83 (2010).

[7] S. Hussain, B. Ahmad, Some properties of soft topological spaces, Comput. Math. Appl., 62, 4058-4067 (2011).

[8] J. Mahanta and P. K. Das, Results on fuzzy soft topological spaces, arXiv:1203.0634v1, (2012).

[9] P. K. Maji, R. Biswas and A. R. Roy, Fuzzy soft sets, J. Fuzzy Math., 9, 589-602 (2001).

[10] P. K. Maji, R. Biswas and A. R. Roy, Intuitionistic fuzzy soft sets , J. Fuzzy Math., 9, 677-692 (2001).

[11] P. K. Maji and A. R. Roy, Soft set theory, Comput. Math. Appl., 45, 555-562 (2003).

[12] D. A. Molodstov, Soft set theory-first results, Comput. Math. Appl., 37, 19-31 (1999).

[13] T. J. Neog, D. K. Sut, G. C. Hazarika, Fuzzy soft topological spaces, Int. J. Latest Trend Math., 2, 54-67 (2012).

[14] S. Roy and T. K. Samanta, A note on fuzzy soft topological spaces, Ann. Fuzzy Math. Inf., 3, 305-311 (2012).

[15] M. Shabir, M. Naz, On soft topological spaces, Comput. Math. Appl., 61, 1786-1799 (2011).

[16] D. K. Sut, An application of fuzzy soft relation in decision making problems, Int. J. Math. Trends Tech., 3, 50-53 (2012).

[17] D. Wardowski, On a soft mapping and its fixed points, Fixed Point Theory Appl. doi:10.1186/1687-1812-2013182, 2013, 182 (2013).

[18] L. A. Zadeh, Fuzzy sets, Inf. Control, 8, 338-353 (1965). 


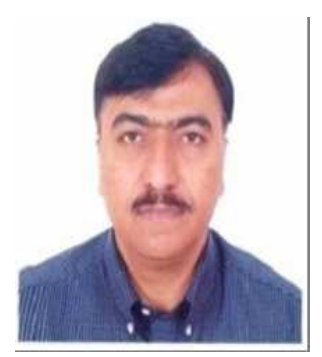

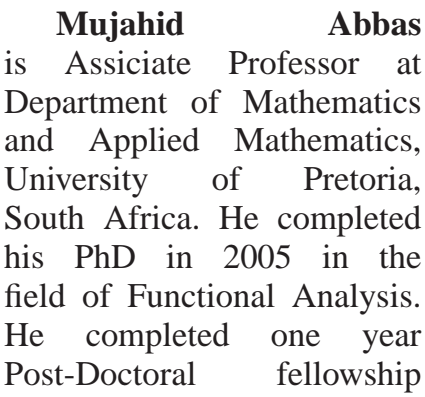

(2006-2007) at Indiana University, Bloomington, U.S.A and one year Post-Doctoral fellowship (2010-2011) at University of Birmingham, United Kingdom. He is working in the field of Fixed-point theory and its applications, Topological vector spaces \& nonlinear operators, Best approximations, Fuzzy logic, and Convex Optimization theory.

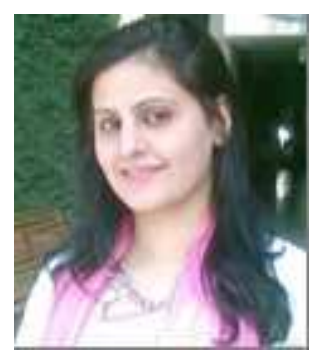

\section{Asma Khalid is a research scholar at} Department of Mathematics, School of Science and Engineering, Lahore University of Manegent sciences, Lahore, Pakistan. Her area of research is Decision Making using Fuzzy Logic. She has published work in the fields of Judgment Aggregation using fuzzy logic based framework, Preference Aggregation and Incomplete Preferences. Her interests embrace Soft Set theory and Fuzzy soft set theory.

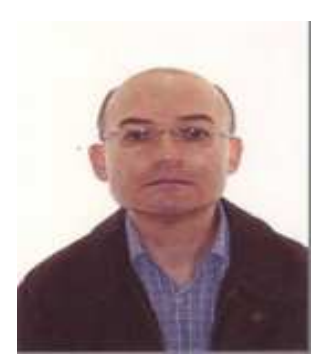

Salvador Romaguera is Full Professor at Department of Applied Mathematics and Institute of Pure and Applied Mathematics, Polytechnic University of Valencia, Spain. His research interests are in the areas of Topology, Fuzzy Mathematics, Fixed Point Theory, and their applications. He has published research articles in several reputed journals of mathematics, applied mathematics and computer science, and is editor and referee of mathematical journals. 